\title{
Spontaneous localization of two kinds of hard spheres in giant vesicles
}

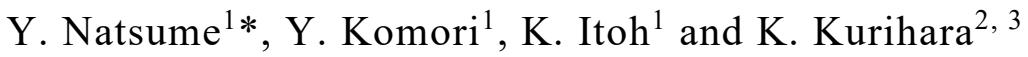 \\ 1 Japan Women's University \\ 2 Exploratory Research Center on Life and Living Systems (ExCELLS) \\ 3 Institute for Molecular Science \\ * Corresponding author: E-mail: natsumey@fc.jwu.ac.jp
}

\begin{abstract}
A phase separation in a dispersion of two kinds of particles has recently been reported to result from entropic interactions between particles and between particles and surfaces in closed spaces. In many cases, the phase separation took place localization of large particles in the vicinity of a compartment. However, phase separation in closed systems has been limited to systems in which the number of small particles was much larger than the number of large particles. In contrast, we prepared giant vesicles (GVs) in which the volume fraction of large particles was higher than that of the small particles. As a result, we observed a new phenomenon in which small particles localized spontaneously and stably in the vicinity of the vesicular membrane. To explain this phenomenon theoretically, we assumed that an equilibrium osmotic pressure was realized between an outer phase containing a relatively large number of small particles and another inner phase. The osmotic pressure was estimated from the free energy change due to the excluded-volume effect. There was good agreement between the distribution ratio of the number of large and small particles in the phases calculated from fluorescent microscopy images and the prediction of the osmotic equilibrium model.
\end{abstract}

Key words: Phase separation, Colloidal particles, Giant vesicles, Osmotic equilibrium

\section{INTRODUCTION}

Phase separation of a system composed of relatively large and small colloidal particles is known to occur by entropic interactions between the particles. Recently, it has become clear that entropic interactions also occur between the particles and the surface of the container. Systems consisting of binary mixtures of hard spheres in closed spaces have also been studied [1]. Phase separation of both large and small colloidal particles has been achieved experimentally in vesicles [2] and water-in-oil emulsions [3]. In each case, phase separations of large particles localized in the vicinity of the boundary membrane were observed if the small particles greatly outnumbered the large particles. However, there have been few reports of experiments involving large particles that were not greatly outnumbered by small particles. We therefore constructed a vesicular system in which both the large and small particles were statistical ensembles by using a water-in-oil emulsion centrifugation method $[4,5]$. We observed stable localization of the small particles in the vicinity of the vesicular membranes.

In traditional work for phase separation of twocomponent solutions or polymer solutions, a lattice model has emerged in which occupation by two molecules of the same lattice is prohibited by the excluded-volume effect.

We thus considered partial differentiation of the free energy change caused by the excluded-volume effect. This treatment corresponds to a statistical explanation of osmotic pressure, as discussed in ref [6]. In the current study, we used the osmotic equilibrium model to explain the novel phase separation of a mixture of particles based on the excluded-volume effect.

\section{EXPERIMENTAL}

2.1 Materials

1, 2-Dioleoyl-sn-glycero-3-phosphocholine (DOPC) was purchased from Avanti Polar Lipids (Alabaster, AL, USA). For the inner microspheres, we purchased non-

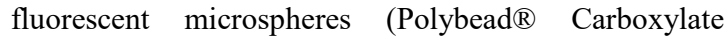
Microspheres) with diameters of either $0.10 \mu \mathrm{m}$ or $1.0 \mu \mathrm{m}$ and fluorescent microspheres with diameters of either 1.0 $\mu \mathrm{m}$ (Fluoresbrite ${ }^{\circledR}$ Yellow Green Microspheres) or 0.10 $\mu \mathrm{m}$ (Fluoresbrite ${ }^{\circledR}$ YO Carboxylate Microspheres) from Polysciences, Inc. (Warrington, PA, USA). Liquid paraffin, D-glucose, and sucrose were purchased from WAKO Pure Chemical Co. (Tokyo, Japan). Tris-HCl buffer solution (1 M, pH 7.5) was provided by Nacalai Tesque, Inc. (Tokyo, Japan). These microspheres were used without further purification.

\subsection{Preparation}

The vesicular dispersion was prepared according to our previously reported protocol [4]. A chloroform solution of DOPC $(25 \mathrm{~mL}, 51 \mu \mathrm{L})$ was evaporated under flowing nitrogen gas. After the addition of liquid paraffin to the DOPC lipid film, the film was incubated at $80{ }^{\circ} \mathrm{C}$ overnight. This oil solution (1.3 mM DOPC) was layered onto the inner aqueous solution $(0.02 \mathrm{M}$ Tris- $\mathrm{HCl}[\mathrm{pH}$ 7.5] and $0.15 \mathrm{M}$ sucrose) containing two kinds of beads (diameters $=0.10 \mu \mathrm{m}$ and $1.0 \mu \mathrm{m}$ ) and then emulsified with a mechanical homogenizer at $10000 \mathrm{rpm}$ for $2 \mathrm{~min}$ at room temperature. The emulsified dispersion was 
layered on the outer phase $(0.02 \mathrm{M}$ Tris- $\mathrm{HCl}[\mathrm{pH} 7.5]$, $0.15 \mathrm{M}$ glucose) and centrifuged at $4{ }^{\circ} \mathrm{C}$ at $9000 \times \mathrm{g}$ for $30 \mathrm{~min}$. The precipitated giant vesicles $(\mathrm{GVs})$ were collected and observed under a fluorescence microscope.

\subsection{Observation}

GVs were observed by using a phase contrast/fluorescence microscope (IX71 Inverted Microscope, Olympus Corp., Tokyo, Japan). Fluorescence microscopy images were obtained with the microscope fitted with U-FMCHE $(\lambda \mathrm{ex}=565-585 \mathrm{~nm}$, $\lambda \mathrm{em}=600-690 \mathrm{~nm})$ and U-FBNA $(\lambda \mathrm{ex}=470-495 \mathrm{~nm}$, $\lambda \mathrm{em}=510-550 \mathrm{~nm}$ ) filter units (Olympus Corp., Tokyo, Japan)

2.4 Estimation of the volume fractions of the smaller microspheres

To estimate the volume fraction of the small microspheres $($ diameter $=0.10 \mu \mathrm{m})$ in the GVs, we used a calibration curve constructed from the concentrations of the small spheres in bulk dispersions. Under conditions in which the fluorescence intensity was not saturated, we experimentally confirmed that the fluorescence intensity was proportional to both the volume density of the fluorescent microspheres and the depth of the dispersion. A calibration curve for small, red microspheres was prepared by plotting the fluorescence intensities of the red microspheres in dispersions containing large and small microspheres. In contrast, the diameters of the GVs and the average values of the red fluorescence of GVs containing large and small microspheres were estimated by using the ImageJ software package. The GV measurement results were applied to the calibration curve for the dispersion, and the volume fraction of each GV was determined. We analyzed $32 \mathrm{GVs}$ and found that $50 \%$ of all volume fractions fell between 0.005 and 0.015 , an interval that included the peak volume fraction of 0.01 . The volume fraction of the small spheres in the GVs was therefore estimated to be 0.01 . The volume fraction of the small spheres was estimated from the concentration of the small spheres in the bulk dispersions that were used for GV preparation.

2.5 Estimation of the volume fractions of the large microspheres

The volume fractions of the large microspheres (diameter $=1.0 \mu \mathrm{m})$ in the GVs were estimated as previously described in our report [5]. The fluorescent microspheres were counted under a fluorescence microscope. The total number $N$ of microspheres encapsulated in the GVs was calculated. The volume fraction $\varphi_{L}$ of the large microspheres in the GVs was determined as follows: $\varphi_{L}=N \times v_{L} / V$, where $v_{L}$ and $V$ are the volume of the larger microsphere and the GV, respectively, and $N$ is the number of encapsulated microspheres in the GV.

\subsection{Estimation of distribution of small particles}

A fluorescence microscope image was taken with an epifluorescence microscope in the focal plane of the maximum cross section of a GV (e.g., Fig. 1b). To determine the number density of the fluorescent particles, the fluorescence intensity of the inner vesicle particles was calculated by subtracting the reference intensity from the fluorescence intensity of the dispersion medium.

\subsection{Estimation of distribution of large particles}

To estimate the distribution of large particles, the focus of the microscope was set in the plane of the maximum cross section of a GV (e.g., Fig. 1c). ImageJ was used to estimate the center of the fluorescent particles and calculate the distance from the center of the vesicle to the center of each particle. The calculated distance was normalized to the diameter of the vesicle.

\section{RESULTS AND DISCUSSION}

3.1 Localization of small spheres in the vicinity of a GV inner membrane wall

Electrostatic interactions due to the surface charge of the polystyrene beads were screened using a buffer solution as the aqueous phase. The Debye-Hückel screening length is on the order of nanometers, and thus the length scale of interparticle electrostatic repulsion was much smaller than that of the observed microscopic-scale events in this study $[7,8]$. We used sucrose to maintain the specific gravity of the inner water phase at 1.05 , which was the same as that of the polystyrene microspheres [sec. 2.2]

In this experiment, gravity and buoyancy therefore had no effect on the microspheres. Consequently, our GV system could be treated as a hard-sphere system. We prepared a DOPC GV system with a defined target volume fraction $\left(\varphi_{L}>\right.$ $\left.\varphi_{s}\right)$ using the water-in-oil emulsion centrifugation method [4, 5]. The sphere diameter ratio was determined to be 10 because phase separation occurs in a binary particle suspension at that ratio $[9,10]$. We observed GVs containing two types of microspheres: $0.1-\mu \mathrm{m}$-diameter spheres, $5 \%$ of which were red-fluorescent spheres; and $1.0-\mu \mathrm{m}-$ diameter spheres, 5\% of which were greenfluorescent spheres [sec. 2.3]. A phase contrast microscope image (Fig. 1a) revealed numerous large spheres encapsulated in the GV. The fluorescence of the small, red-fluorescent spheres increased at the edge of the GV (Fig. 1b). Large, green-fluorescent spheres of the large particles were observed with spherical fluorescence (Fig. 1c). Figure 1d, a merged image of Fig. $1 \mathrm{~b}$ and Fig. $1 \mathrm{c}$, shows that small and large spheres were contained in the same GV. The red fluorescence in the image (Fig. 1e) was bright in the vicinity of the GV membrane and relatively dark elsewhere.

In a control experiment, a preparation of GVs containing only small spheres was incubated at $4{ }^{\circ} \mathrm{C}$ for $4 \mathrm{~h}$ prior to observation by phase contrast microscopy (Fig. 1f) and fluorescence microscopy (Fig. 1g). The fluorescence microscope image showed many small spheres encapsulated in the GV. Figure $1 \mathrm{~h}$ shows the intensity of fluorescence derived from the red microspheres. The 
The GV containing two kinds of microspheres

(a)

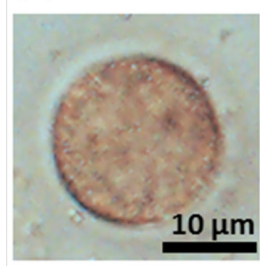

(b)

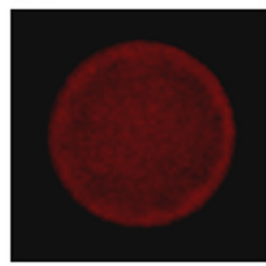

(c)

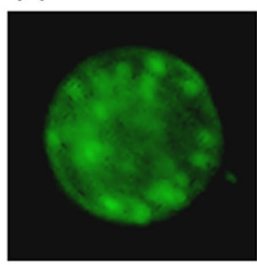

(d)

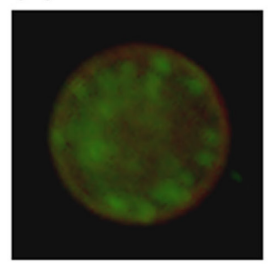

(e)

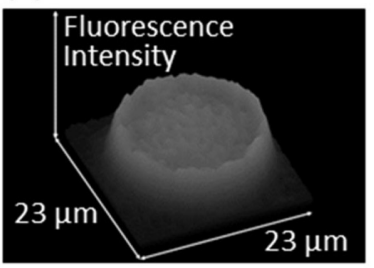

The GV containing only small microspheres

(f)

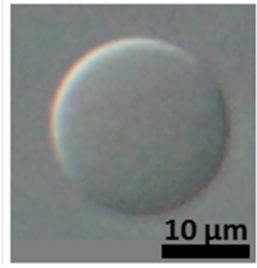

(g)

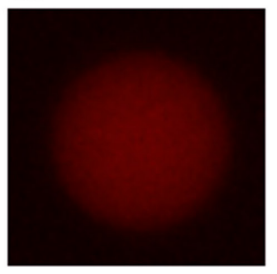

(h)

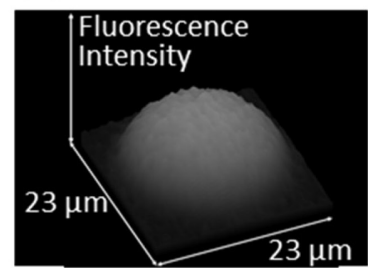

Figure 1. Phase difference and fluorescence microscopy images and distribution of fluorescence intensity. (a)-(d) Microscopy images of a GV containing two kinds of microspheres. (a) Phase difference microscopy image, (b) fluorescence microscopy image of small spheres dyed red, (c) fluorescence microscopy image of large spheres dyed green, and (d) stacked image of Fig. 1(b) and Fig. 1(c). (e) Surface plot of the fluorescence intensity image of Fig. 1(b) showing that small spheres were localized in the vicinity of the GV membrane. In contrast, (f)-(h) are images of the GV containing only small microspheres. (f) Phase difference microscopy image and (g) fluorescence microscopy image of a GV containing only small red fluorescent microspheres and (h) surface plot of the fluorescence intensity image of Fig. 1(g). In contrast to (e), fluorescence intensity decayed smoothly toward the GV membrane.

fluorescence intensity was higher near the center of the GV and decayed smoothly toward the GV membrane due to the higher fluorescence intensity in the thicker part of the sample.

These results showed that only the small spheres were uniformly dispersed inside the GV, and thus the uneven distribution (localization) observed in the mixed-sphere systems must have been due to the small spheres in the vicinity of the GV membrane. This phenomenon was observed 50 times. In our system, the volume fraction $\varphi_{s}$ was estimated to be 0.01 [sec. 2.4], whereas the volume fraction $\varphi_{L}$ was different in each GV [sec. 2.5]. The average $\varphi_{L} / \varphi_{S}$ ratio was 6.4 , and the standard deviation was 3.3 .

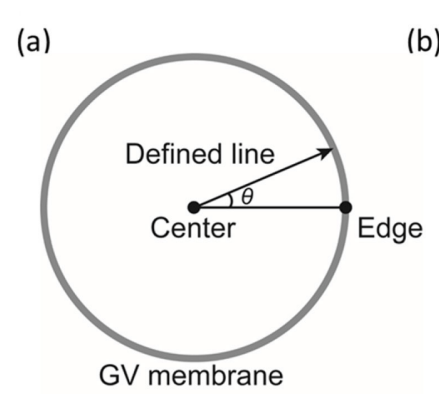

3.2 Time series observation of vesicle exhibiting localization

To evaluate the stability of the localization of the small microspheres, we analyzed the temporal changes of the red fluorescence intensities of the small spheres. We drew an arbitrarily defined line from $10^{\circ}$ to $360^{\circ}$ at intervals of $10^{\circ}$ on the microscopy image of fluorescence at 24 and $48 \mathrm{~h}$ after preparation of the GVs (Fig. 2a). We compared the values of fluorescence intensity on the arbitrarily defined lines for both graphs. Figure $2 b$ shows the average fluorescence intensities on lines from $0^{\circ}$ to $360^{\circ}$ in the images of the GVs at 24 or $48 \mathrm{~h}$ after preparation. The intensities were normalized so that the average over all angles was 100. In both cases, the difference between the normalized intensities of the images of the GV at 24 and $48 \mathrm{~h}$ was $<5 \%$ at all angles. This result means that

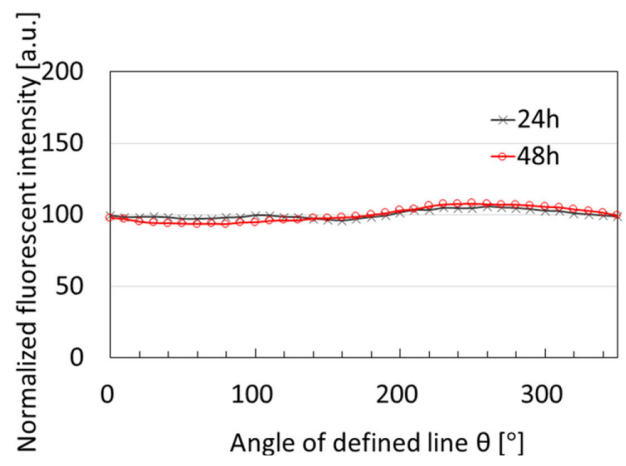

Figure 2. (a)Schematic image of angle of defined line. Fluorescent intensities of vesicles were calculated every 10 degree. (b)Comparison of the fluorescence intensity of the images of the vesicles 24 and $48 \mathrm{~h}$ after preparation. Because the fluorescence intensities at 24 and $48 \mathrm{~h}$ at the same position of the vesicle were very similar, localized small particles were stable over time. 
there was no difference in the distributions of fluorescence intensities at 24 and $48 \mathrm{~h}$. This result indicates that the distribution of red fluorescence intensity remained unchanged during the fluorescent microscopic observations; i.e., the localization of the small spheres was stable. This result shows that small sphere localization had reached an equilibrium by $24 \mathrm{~h}$.

\subsection{Osmotic equilibrium model}

To account for the localization of small particles in the GVs, we envisioned an osmotic equilibrium model and introduced a hypothetical boundary between phase A (inner spherical region of GV) and phase $\mathrm{B}$ (region between vesicular membrane and phase A) (Fig. 3). We calculated the osmotic pressure by partially differentiating the free energy change $\Delta \mathrm{G}$ with respect to volume owing to the excludedvolume effect.

Generally, in a closed space of volume $V$ containing $N$ particles, each of which has a volume $v$, the volume fraction $\varphi$ is $\varphi=\frac{N v}{V}$. The free energy change $\Delta G$ is

$$
\Delta G=-k_{B} T \log \left(\frac{Z^{\text {ideal }}}{Z^{\text {exc }}}\right),
$$

using the partition function $Z$ [6].

Therefore, the osmotic pressure $P$ is given by

$$
P=-\frac{\partial G}{\partial V}=\frac{N k_{B} T}{V} \frac{N v}{V}
$$

The two phases (phase A and phase B) satisfied the balanced stress condition owing to the excluded-volume interaction. The pressures of both phases $\left(P_{A}\right.$ and $\left.P_{B}\right)$ containing both large and small particles were estimated as follows. Inside the GV, the total numbers of large and small particles were assumed to be $N_{L}$ and $N_{S}$, respectively Thus, the numbers of large and small

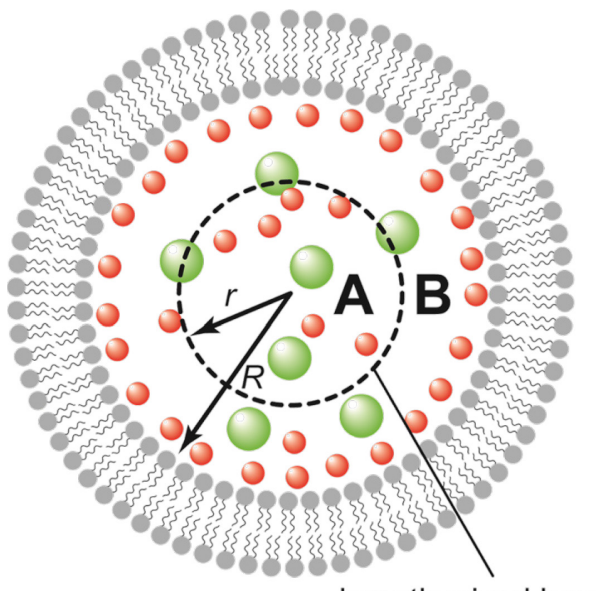

hypothesized boundary

Figure 3. Schematic illustration of osmotic equilibrium model. We hypothesized the boundary (dashed circle) for the model and assumed that the osmotic pressures of phase A and phase B were equal. Phase $A$ is the spherical space between the center and supposed boundary. Phase B is the small particles excess area between the hypothesized boundary and the vesicular membrane. $R$ is the radius of the vesicle, and $r$ is the distance between the center and the boundary. particles in phase A were assumed as $N_{L}^{A}$ and $N_{S}^{A}$, respectively, and the numbers of large and small particles in phase B were assumed to equal $N_{L}^{B}$ and $N_{S}^{B}$, respectively. According to Eq. 2, the osmotic pressure of each phase may be expressed as follows.

$$
\begin{aligned}
& P_{A}=-\frac{\partial \Delta G_{A}}{\partial V^{A}}=\frac{N_{L}^{A} k_{B} T}{V_{A}} \frac{N_{L}^{A} v_{L}}{V_{A}}+\frac{N_{S}^{A} k_{B} T}{V_{A}} \frac{N_{S}^{A} v_{S}}{V_{A}} \\
& P_{B}=-\frac{\partial \Delta G_{B}}{\partial V^{B}}=\frac{N_{L}^{B} k_{B} T}{V_{B}} \frac{N_{L}^{B} v_{L}}{V_{B}}+\frac{N_{S}^{B} k_{B} T}{V_{B}} \frac{N_{S}^{B} v_{S}}{V_{B}}
\end{aligned}
$$

where $V_{A}$ and $V_{B}$ are the volumes of phases A and $\mathrm{B}$, respectively. If $R$ is the radius of the $\mathrm{GV}$ and $r$ is the radius of phase $\mathrm{A}$, volumes $V_{A}$ and $V_{B}$ may be expressed as $V_{A}=\frac{4}{3} \pi r^{3}$ and $V_{B}=$ $\frac{4}{3} \pi\left(R^{3}-r^{3}\right)$, respectively. Under the condition that the osmotic pressure of phase $A$ is equal to that of phase B, we get the boundary between the phases $\mathrm{A}$ and $\mathrm{B}$.

\subsection{Application of equilibrium model to experiments}

To explain the experimental results, the following analysis was carried out on the basis of the equilibrium model. First, from the fluorescence microscope images, the ratio of the numbers of small and large particles in both phase A and phase $B$ was calculated. Second, by using these ratios and the total numbers of small and large particles in the GVs, the numbers $\left(N_{L}^{A}, N_{L}^{B}, N_{S}^{A}, N_{S}^{B}\right)$ of the two kinds of particles in each phase were obtained. Third, these values were substituted into eq. 3 and eq. 4 to obtain the osmotic pressure of each phase.

For calculating the ratio of the two kinds of particles, the fluorescence microscopy images of Fig. 1(b) and (c) (radius: $9.7 \mu \mathrm{m}, \varphi_{S}=0.01$, $\left.\varphi_{L}=0.063\right)$ were analyzed. The ratios of the particles in phase A and phase $\mathrm{B}\left(n_{S}^{B} / n_{S}^{A}, n_{L}^{B} / n_{L}^{A}\right)$ were determined from the fluorescence microscopy images in two-dimension. The distribution of small particles was estimated from Fig. 1(b). The dependence on the radius $r$ of fluorescence intensity was obtained by adding fluorescence intensities for any direction on the plane of the maximum cross section of the GV. The obtained result is shown as a black line in Fig. 4. Here, the contribution from the background was subtracted. Further, each of the intensities were multiplied by $2 \pi r$ to obtain their distribution on a circular ring with width $\Delta r=R / 45$. By the use of these corrected intensities, the rate of $n_{S}^{B} / n_{S}^{A}$ was obtained for any boundary $r / R$.

From Fig. 1(c), the center spots of large particles in the GV were determined to calculate the ratio $n_{L}^{B} / n_{L}^{A}$ by using ImageJ (sec. 2.7).

In fact, the boundary for the model (i.e., input radius $r / R$ ) was adopted for $r / R=0.3-0.8$. To determine $N_{L}^{A}, N_{L}^{B}, N_{S}^{A}$, and $N_{S}^{B}$, we derive the ratios $N_{S}^{B} / N_{S}^{A}$ and $N_{L}^{B} / N_{L}^{A}$. By using $n_{S}^{B} / n_{S}^{A}$ and $n_{L}^{B} / n_{L}^{A}$ for any input radius $r / R$ as for a twodimensional disk, we could obtain $N_{S}^{B} / N_{S}^{A}$ and 
$N_{L}^{B} / N_{L}^{A}$ in the GV adopting the correction function that was given for each radius $r / R^{*}$. Following this procedure, we obtained $N_{L}^{A}, N_{L}^{B}, N_{S}^{A}$ and $N_{S}^{B}$, as we had estimated the total number $N_{S}$ and $N_{L}$ in the $\mathrm{GV}^{* *}$. Substituting these values in eq. 3-4, we obtained osmotic pressures $P_{A}$ and $P_{B}$ as illustrated by the red and blue dash lines in Fig. 4.

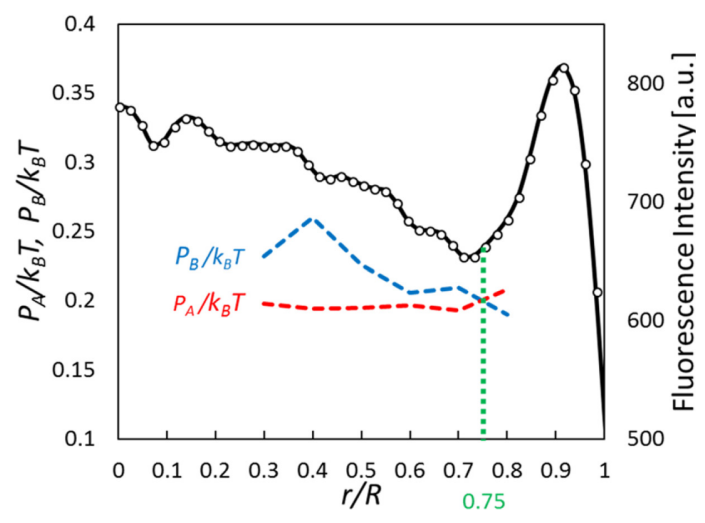

Figure 4. The black solid line represents the fluorescence intensity of small particles for the radial direction $r / R$. Osmotic pressures $P_{A}$ and $P_{B}$ are plotted in units of $k_{B} T$ as the function of the radius $r / R$. The procedure for estimation of the osmotic pressure from this black line is described in the text. As shown by the green dotted line, the intersection is found at 0.75 , at which the osmotic pressure of phase $\mathrm{A}$ is equal to that of phase $\mathrm{B}$.

Assuming values of $r / R$ ranging from 0.3 to 0.8 , the above analysis was carried out to calculate $P_{A}$ and $P_{B}$ (Fig. 4). The hypothetical boundary $r / R$ was taken as $\mathrm{x}$ variable and the calculated $P_{A}$ and $P_{B}$ as the y variables. As clearly shown, in the case of $r / R=0.75$, the osmotic pressure of phase A was equal to that of phase $B$. In the case where $r / R=0.75, N_{S}^{B} / N_{S}^{A}=1.3$ and $N_{L}^{B} / N_{L}^{A}=0.98$. The values of $N_{S}^{A}, N_{S}^{B}, N_{L}^{A}$, and $N_{L}^{B}$ were 31520,41040 , 233 , and 228 , respectively.

These results lead to the following conclusion: when the hypothetical boundary was located approximately about $75 \%$ from the center to the membrane of $\mathrm{GV}$, the osmotic pressure reaches equilibrium between the small particle-rich phase and the other phase. This theoretical result is consistent with the experimental fact that the small particles are localized stably in the vicinity of the GV by the aid of the distribution of large particles.

\footnotetext{
* As described in sec. 2.3, our fluorescence microscopy images were obtained by epi-illumination. Our data were the particle numbers $n_{A}$ and $n_{B}$ that were contained in the inner part and outer part, respectively, of a disk with some thickness $d$. Thus, we could calculate the ratio between particle numbers $N_{A}$ and $N_{B}$ from the ratio between $n_{A}$ and $n_{B}$, supposing that particles in both regions $\mathrm{A}$ and $\mathrm{B}$ were distributed uniformly with densities of $n_{A}$ and $n_{B}$ in the disk. In short, we could adopt the following correction function, which is independent of
}

\section{CONCLUSION}

We discovered new distribution patterns in giant vesicles containing two kinds of colloidal particles. We demonstrated the localization of small particles in the vicinity of the vesicular membrane for systems where large particle have a relatively large amount of volume fraction. Furthermore, we analyzed the localization on the basis of the osmotic equilibrium model. The analysis reveals phase separation at a certain boundary, which is in agreement with the experimental results. It should be noted that the localization of small particles occurs by the thermal statistics (osmotic) effect of the large particles. In fact, the osmotic pressure comprises the contribution of both the large and small particles.

As reported in previous studies, an overwhelming majority of small particles lead to a depletion force between the large particles and the membrane [11]. In contrast, in the current study, the large particles serve as a thermal statistical ensemble. The attractive depletion force between the small particles and GV membrane occurs due to the large particles. This aspect was elucidated the first time. Thus, the present experimental results may be more clearly explained on the basis of our phenomenological model in which effective repulsive interaction between particles is taken into account by the excluded-volume effect.

\section{REFERENCES}

[1] A. G. Yodh, K-H. Lin, J. C. Crocker, A. D. Dinsmore, R. Verma and P. D. Kaplan, Phil Trans $R$ Soc Lond A, 359, 921-937(2001).

[2] A. D. Dinsmore, D. T. Wong, P. Nelson and A. G. Yodh, Phys Rev Lett, 80, 409-412(1998).

[3] G. Meng, J. Paulose, D. R. Nelson and V. N. Manoharan, Science, 343, 634-637(2014).

[4] Y. Natsume, H-I Wen, T. Zhu, K. Itoh, L. Sheng and $\mathrm{K}$. Kurihara, $J$ Vis Exp, 119, 55282(2017).

[5] Y. Natsume and T. Toyota, Chem Lett, 42, 295297(2013)

[6] R. Phillips, J. Kondev, J. Theriot and H. G. Garcia, Physical Biology of the Cell, Garland Science, USA, second edition, chapter 14, 543571(2012).

[7] A. D. Dinsmore, D. T. Wong, P. Nelson and A. G. Yodh, Phys Rev Lett, 80, 409-412(1998).

[8] P. D. Kaplan, J. L. Rouke, A. G. Yodh and D. J. Pine, Phys Rev Lett, 72, 582-585(1994).

[9] A. D. Dinsmore, A. G. Yodh and D. J. Pine, Phys Rev E, 52, 4045-4057(1995).

$d:$

$$
\frac{N_{B}}{N_{A}}=\frac{n_{B}}{n_{A}} \times \frac{1-\left(\frac{r}{R}\right)^{3}}{1-\left(\frac{r}{R}\right)^{2}} \times \frac{R}{r}
$$

** Here, the total number of small particles and the total number of large particles in the GV can be obtained from the volume fraction of large and small particles (sec. 2.3, 2.4), and the diameter of the GV can be obtained from the red fluorescent microscopy image. 
[10] H. Furusawa and M. Inoue, Intell Autom Soft Co, 18, 187-199(2012).

[11] H. N. W. Lekkerkerker and R. Tuinier, Colloids and the Depletion Interaction, Springer, Germany, chapter 2, 57-108(2011).

\section{ACKNOWLEDGEMENTS}

We thank Prof. T. Toyota (The University of Tokyo) for valuable discussions. This study was supported by a Grant-in-Aid for Young Scientists (B) (to Y.N., no. 17K14374) and a Grant-in-Aid for Young Scientists (A) (to K.K., no. 17H04876) from the Japan Society for the Promotion of Science; by the Sasakawa Scientific Research Grant from The Japan Science Society (to Y.N., no. 29-217).

(Received January 31, 2018; Accepted October 1, 2018;

Published Online December 1, 2018) 